\title{
Clinical Spectrum of Chronic Lower Gastrointestinal Bleeding in Sub-Himalayas: A Study at a Tertiary Care Hospital of North India
}

\author{
Vikas Lakhanpal ${ }^{1}$ Rajesh Sharma ${ }^{2}$ Vishal Bodh² \\ ${ }^{1}$ Department of Medicine, Indira Gandhi Medical College, Shimla, \\ Himachal Pradesh, India \\ 2Department of Gastroenterology, Indira Gandhi Medical College, \\ Shimla, Himachal Pradesh, India \\ ${ }^{3}$ Department of Physiology, Indira Gandhi Medical College, Shimla, \\ Himachal Pradesh, India
}

J Digest Endosc 2019;10:158-162

\author{
Surinder Thakur ${ }^{1} \quad$ Neetu Sharma ${ }^{3}$ Brij Sharma
}

\author{
Abstract, \\ Keywords \\ - lower gastrointestinal \\ bleeding \\ - inflammatory bowel \\ disease \\ - solitary rectal ulcer \\ syndrome \\ - colonoscopy
}

\author{
Address for correspondence Brij Sharma, MD, DM, Department \\ of Gastroenterology, Indira Gandhi Medical College, Ridge \\ Sanjauli Rd, Lakkar Bazar, Shimla 171001, Himachal Pradesh, India \\ (e-mail: drbrijsharma01@gmail.com).
}

Background Lower gastrointestinal bleeding (LGIB) is fairly a common presentation in gastroenterology and medicine departments, and presents major diagnostic and therapeutic challenges. This study aimed to explore the varied clinical spectrum of LGIB in our region.

Methods A total of 138 patients presented with chronic LGIB and were investigated using colonoscopy and relevant investigations.

Results The majority of patients were in the age group of 40 to 59 years, with a mean age of 49.5 years, slight male predominance, and male-to-female ratio of 1.19:1. The most common clinical presentation was hematochezia (97.8\%). Overall, $15 \%$ patients had more than one comorbidity, and $39.13 \%$ patients were anemic, of which $7.24 \%$ received blood transfusions. Diagnostic yield of colonoscopy was $92.75 \%$.

Major causes of LGIB were anorectal causes (19.56\%), inflammatory bowel disease (19.56\%), colorectal carcinoma (17.39\%), radiation proctitis $(9.42 \%)$, infective causes (11.59\%), nonspecific colitis (7.24\%), and benign growths (5.07\%). However, in the elderly (age $>60$ years), carcinoma colon, radiation proctitis, and hemorrhoids predominated the clinical picture.

Conclusion Colonoscopy is very useful in the evaluation of patients with LGIB. The predominant causes of LGIB vary according to age as well as geographical location, and mortality of LGIB is negligible as compared with upper gastrointestinal bleeding.

\section{Introduction}

Lower gastrointestinal bleeding (LGIB) is anatomically defined as bleeding beyond the ligament of Treitz. ${ }^{1,2}$ It encompasses a wide spectrum of disease, ranging from occult gastrointestinal bleeding to hematochezia to life-threatening bleeding, with an estimated annual incidence of hospitalization to be around 20 to 30 per 100,000 persons. ${ }^{2}$ Studies on LGIB is less as compared with those on upper gastrointestinal bleeding. As compared with patients with upper gastrointestinal bleeding, those with LGIB are less likely to experience shock, have higher hemoglobin levels, often require fewer blood transfusions, ${ }^{3}$ and have favorable outcomes despite comorbidities and old age. ${ }^{4}$ Overall mortality from LGIB ranges from 2 to $4 \% .^{5}$

There are worldwide regional differences in the causes of LGIB. In Western Europe and the United States, diverticulitis coli is common and also one of the most common causes of LGIB. ${ }^{1}$ In Asia, however, diverticulitis coli is a much less common cause of LGIB. Various population-based studies suggest that prevalence of diverticular disease is 17 to $40 \%$, colonic vascular ectasia 2 to 30\%, colitis (ischemic, infectious, 
inflammatory bowel disease [IBD], radiation proctitis) 9 to $21 \%$, colonic neoplasia/postpolypectomy bleeding 11 to $14 \%$, anorectal causes 4 to $10 \%$, upper gastrointestinal sites 0 to $11 \%$, and small bowel sites including Crohn's ileitis, vascular ectasia, Meckel's diverticulitis, and tumors 2 to $9 \%$. $^{6-8}$

The most common etiologies of LGIB vary according to the age of the patients. In adults aged less than 60 years, the most frequent source of LGIB includes colonic diverticula and IBD, whereas angiodysplasia, diverticular disease, neoplasms, and ischemia are the most common causes of LGIB in the elderly. ${ }^{2}$ As in upper gastrointestinal bleeding, patients who begin bleeding while hospitalized for a separate disease process (inpatient bleeding) have a significantly higher risk of death than those who are admitted with LGIB (23 vs. $2.4 \%$ ). ${ }^{2}$ Mortality due to LGIB is largely attributed to the etiological cause and coexistent comorbidities rather than the direct result of uncontrolled bleeding. ${ }^{2}$ Studies have shown that colonoscopy, particularly when performed early (within 12-24 hours of admission), is safe and effective, ${ }^{9}$ with the diagnostic yield of colonoscopy ranging from 45 to $95 \% .^{10}$ Early endoscopy should be performed within 24 hours of initial presentation of acute LGIB, if possible. Various colonoscopy treatment procedures include injection, laser coagulation electrocautery, and heater probe. Intravenous fluids, blood transfusion and use of vasoconstrictor agents constitute a few supportive treatment modalities, but management depends on the underlying etiology. Some patients who continue to bleed or having rebleeding may require surgical intervention.

There is a paucity of studies exploring the clinical spectrum of LGIB from the Indian subcontinent especially from north India. Henceforth, this study was conducted at a tertiary care hospital at Shimla in the Departments of Medicine and Gastroenterology.

\section{Aims and Objectives}

The aim of this study is to assess the clinical profile and outcome in patients presenting with LGIB in the Departments of Medicine and Gastroenterology.

\section{Materials and Methods}

A total of 138 patients ( $>60$ years $[n=41]$ and $<60$ years [ $n=97]$ ) who presented to the Departments of Gastroenterology and
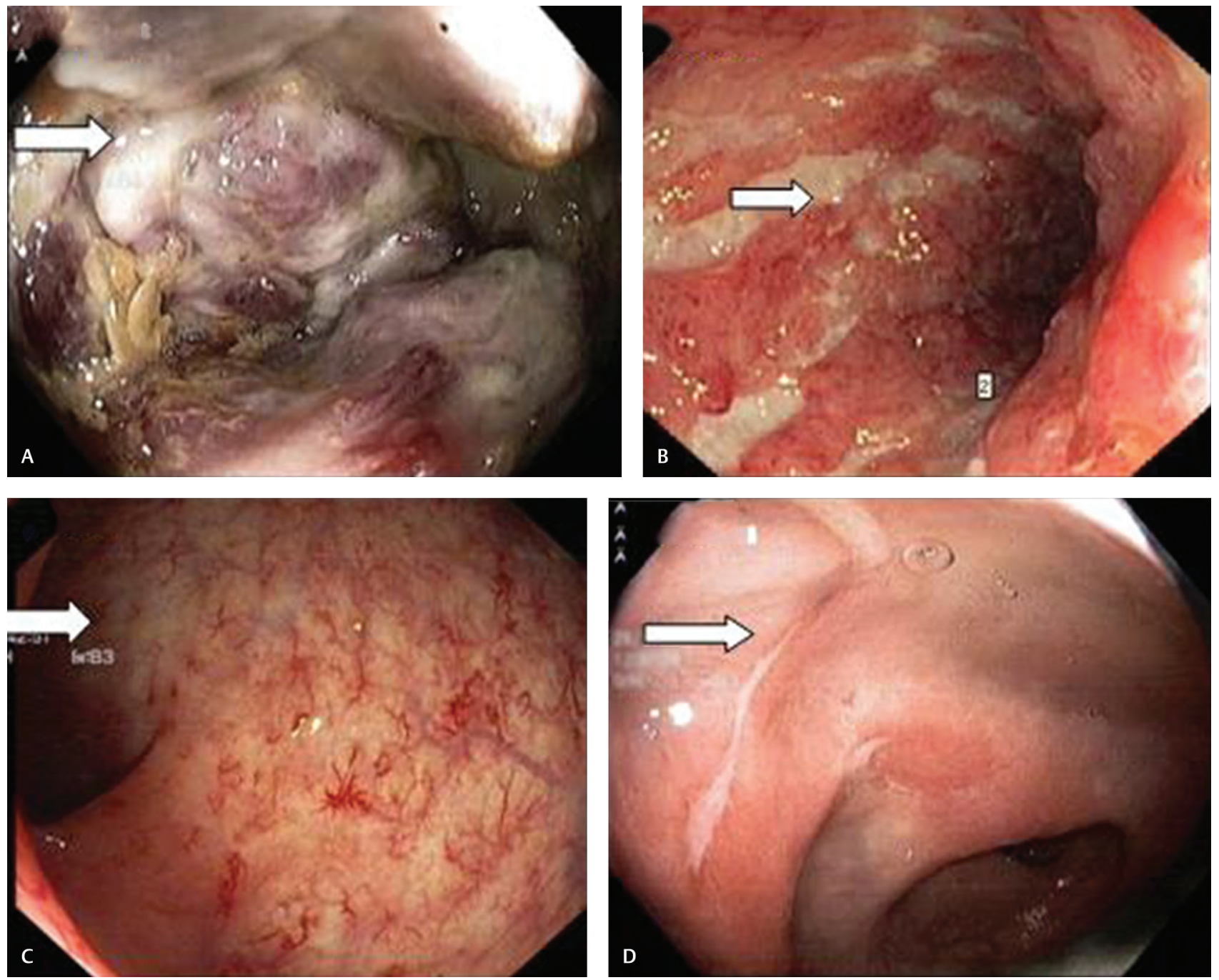

Fig. 1 (A-D) Colonoscopic images of various causes of Lower Gl bleed. 
Medicine were included in the study. After informed consent, physical examination, and routine investigations, all patients were subjected to fibro-optic colonoscopy. Colonoscopic examination was performed after bowel preparation with an Olympus EXERA II CF-H180 AL/I video colonoscope with an outer diameter of $12.8 \mathrm{~mm}$. The bowel was prepared with two packets (each packet of $137.15 \mathrm{~g}$ ) of PEG (polyethylene glycol) -3350 in $4 \mathrm{~L}$ of water, 4 hours before the colonoscopy. Effective bowel cleansing is seen in $90 \%$ of patients (on the first bowel preparation). Patients with inadequate bowel preparation during the first procedure were given repeat preparation with a similar regimen of PEG 3350. Histopathological examination was performed, whenever necessary, for diagnostic purposes.

\section{Results}

The study group comprised 75 (54.34\%) males and 63 (45.65\%) females, with a ratio of 1.19:1. Majority of the patients were in the age group of 40 to 59 years, with all patients falling in the range of 18 to 86 years. Hematochezia was a major presentation of LGIB (97.8\%).

Pain in the abdomen was present in $22.46 \%$ of patients, and majority of the patients were having IBD and radiation proctitis reflecting inflammatory origin of pain. More than one comorbidity was seen in $15 \%$ of patients. Anemia was present in $39.13 \%$, and only $7.2 \%$ patients received blood transfusions.

Colonoscopy was performed in all patients, and the diagnostic yield of colonoscopy was $92.75 \%$. Colonoscopy showed growth colon ( - Fig. 1A), IBD ( - Fig. 1B), telangiectasia of rectum suggestive of radiation proctitis ( - Fig. 1C), and solitary rectal ulcer syndrome (SRUS; - Fig. 1D). None of the patients had severe LGIB, and there was no mortality during hospital stay due to LGIB. In patients whose colonoscopy was normal, computed tomography (CT) scan of the abdomen and CT angiography were also performed, which were normal.

The most common causes of LGIB were anorectal causes (19.56\%) and IBD (19.56\%) followed by colorectal carcinoma (17.39\%), radiation proctitis $(9.42 \%)$, infective causes
(11.59\%), and nonspecific colitis (7.24\%). Benign growths (5.07\%) represented only a few cases of LGIB.

There was also a difference in the etiology of LGIB according to age. In age group less than 60 years, hemorrhoids (20.61\%), IBD (23.71\%), and colorectal carcinoma (14.43\%) dominated the clinical picture, with infective causes (9.27\%) and nonspecific colitis (10.3\%) being minor cases, whereas in the elderly population, colorectal carcinoma (24.39\%), hemorrhoids (17.01\%), and radiation proctitis (19.51\%) dominated the clinical picture ( $\boldsymbol{- T a b l e s} \mathbf{1}$ and $\mathbf{2}$ ).

\section{Discussion}

LGIB represents a diverse range of bleeding sources and severities, ranging from scant bleeding due to hemorrhoids to massive bleeding mainly from vascular sources. There is a considerable difference in the age of presentation of LGIB in the eastern and western part of India. The annual incidence

Table 1 Clinical profile of patients with lower gastrointestinal bleeding $(n=137)$

\begin{tabular}{|l|l|l|}
\hline Clinical characteristics & $\mathbf{n = 1 3 8}$ & Percentage \\
\hline Age $<60$ y & 97 & 70.28 \\
\hline Age $>60$ y & 41 & 29.71 \\
\hline Males & 75 & 54.34 \\
\hline Females & 63 & 65.65 \\
\hline Painless bleeding & 100 & 72.46 \\
\hline Painful bleeding & 35 & 25.36 \\
\hline $\begin{array}{l}\text { Stool with occult blood } \\
\text { positive }\end{array}$ & 2 & 1.44 \\
\hline Stool mixed with blood & 1 & 0.72 \\
\hline Anemia & 54 & 39.13 \\
\hline $\begin{array}{l}\text { Blood transfusion } \\
\text { required }\end{array}$ & 10 & 7.2 \\
\hline Pain abdomen & 31 & 22.46 \\
\hline Drug intake history & 3 & 3 \\
\hline
\end{tabular}

Note: diagnostic yield of colonoscopy is $92.75 \%$.

Table 2 Etiological profile of lower gastrointestinal bleeding

\begin{tabular}{|l|l|l|l|}
\hline Etiology behind LGIB & $\boldsymbol{n}=138$ & Age less than $60 \mathrm{y}(\boldsymbol{n}=\mathbf{9 7})$ & Age greater than $60 \mathrm{y}(\boldsymbol{n}=\mathbf{4 1})$ \\
\hline Hemorrhoids & $27(19.56 \%)$ & $\begin{array}{l}20(20.61 \%) \\
3 \text { (hemorrhoids plus infective } \\
\text { etiology) }\end{array}$ & $\begin{array}{l}7(17.01 \%) \\
1 \text { (hemorrhoids plus benign } \\
\text { growths) }\end{array}$ \\
\hline $\begin{array}{l}\text { IBD (ulcerative colitis and } \\
\text { Crohn's disease) }\end{array}$ & $27(19.56 \%)$ & $4(9.75 \%)$ \\
\hline Carcinoma colon & $24(17.39 \%)$ & $14(14.43 \%)$ & $10(24.39 \%)$ \\
\hline Radiation proctitis & $13(9.42 \%)$ & $5(5.15 \%)$ & $8(19.51 \%)$ \\
\hline Infective etiology & $16(11.59 \%)$ & $9(9.27 \%)$ & $4(9.75 \%)$ \\
\hline Benign growths & $7(5.075 \%)$ & $5(5.15 \%)$ & $1(2.43 \%)$ \\
\hline Nonspecific colitis & $10(7.24 \%)$ & $10(10.3 \%)$ & 0 \\
\hline SRUS & $4(2.89 \%)$ & $2(2.061 \%)$ & $2(4.86 \%)$ \\
\hline Normal study & $10(7.24 \%)$ & $6(6.18 \%)$ & $4(7.75 \%)$ \\
\hline
\end{tabular}

Abbreviations: IBD, inflammatory bowel disease; LGIB, lower gastrointestinal bleeding; SRUS, solitary rectal ulcer syndrome. 
rate of hospitalization increases from 1 per 100,000 patients in the third decade of life to more than 200 per 100,000 in patients in the ninth decade of life. ${ }^{2}$ In the Indian context, LGIB is a disease of comparatively younger population with a slight male predominance (1.19:1, in this study) as compared with the western population. This reflects different etiological patterns in our region compared with the west, as IBDs having their onset usually between 15 and 40 years of age, and hemorrhoidal bleeding is more common in the younger population ( $<60$ years of age) and contributes to a majority of cases as compared with the west.

Most patients with LGIB usually present with hematochezia; however, a significant number of patients present with anorectal bleeding and bloody diarrhea. No definitive studies exist to exactly quantify the magnitude of different presentations. The most common clinical presentation of LGIB in our patients was hematochezia (135/138 [97.8\%]). Pain in the abdomen was present in $22.46 \%$ patients in which more than sixty percent of patients were contributed by IBD (ulcerative colitis), radiation proctitis, and infective etiology, clearly reflecting inflammatory origin of pain. Only three patients had a history of drug intake, reflecting that drug intake is not a major contributor to pain. Compared with western studies in which coexisting comorbid conditions were present in around $70 \%$ of patients, ${ }^{1,11} 15 \%$ of patients in this study had more than one comorbidity, which could be attributed to comparatively younger age of patients.

Patients with LGIB are less likely to experience shock, require fewer blood transfusions, and have significantly higher hemoglobin levels as compared with upper gastrointestinal bleeding. ${ }^{4}$ In our study, $39.13 \%$ patients were anemic, only $7.24 \%$ patients received blood transfusions, and hemodynamic instability was present in $7.97 \%$ patients. In this study, colonoscopy was performed in all patients, and the cause of LGIB could be established in $92.75 \%$ patients, which confirmed the usefulness of early colonoscopy in managing LGIB.

According to Boley et $\mathrm{al}^{12}$ the spectrum of sources appears to be changing over time. In the earlier 20th century, neoplasia was reported as the predominant cause of LGIB, and diverticular disease was rare. Currently, diverticular bleeding is the leading cause of LGIB in developed countries. Colonoscopy is the initial and most common investigation used in the evaluation of Lower GI bleed. ${ }^{13}$ According to Longstreth, ${ }^{2}$ advancing age may be a factor that could be implicated in the cause of this changing trend. Low-fiber diet, better access to nonsteroidal anti-inflammatory drugs, obesity, physical inactivity, hypertension, ischemic heart disease, chronic renal insufficiency, and hyperlipidemia have been proposed to be responsible for the high prevalence of diverticular disease in western countries. In this study, anorectal causes (19.56\%) (hemorrhoids, SRUS), and IBD (19.56\%) (ulcerative colitis) outscored all other causes followed by colorectal carcinoma (17.39\%), radiation proctitis $(9.42 \%)$, infective causes (11.59\%), and nonspecific colitis (7.24\%). Benign growths $(5.07 \%)$ were found only in a minority of patients. Hence, our results are at variance with the experience from the west, where diverticulitis and vascular ectasias are common causes of LGIB, which could be attributed to the traditional Indian diet with high-fiber content. However, recently, there have been reports of the emergence of diverticulitis and colonic polyps in the urban Indian population, ${ }^{10}$ possibly as a result of changing Indian lifestyle. The striking high percentage of radiation proctitis $(9.42 \%)$ in our study is related to the high prevalence of carcinoma cervix and high-dose radiation given to these patients and referral to the radiotherapy center at our hospital. In our study, $11.59 \%$ of patients were attributed to infective causes, in which nonspecific infective ulcers (43.75\%), tubercular pathology (31.25\%), and enteric ulcers $(25 \%)$ are the causes. Similar studies by Goenka et a ${ }^{14}$ and Khandelwal ${ }^{15}$ also showed that infective causes contributed significantly in our part of the world. Tuberculosis is highly prevalent in India and should be considered as an important cause of LGIB in India.

The prevalence of the most common etiologies of LGIB also varies according to the age of the patients. ${ }^{16}$ In patients aged less than 60 years, anorectal causes (hemorrhoids and SRUS), IBD (ulcerative colitis), and colorectal carcinoma dominated the clinical picture followed by radiation proctitis, infective causes, and nonspecific colitis, which contributed to the small percentage of cases. However, the clinical etiology is different in patients aged over 60 years, in whom colorectal cancer and radiation proctitis were major causes followed by hemorrhoidal bleeding, IBD, and benign growths.

Most patients with LGIB have favorable outcomes despite advanced age and comorbid conditions. ${ }^{17}$ Mortality due to LGIB is not the result of bleeding but rather exacerbation of an underlying disorder or development of a nosocomial complication. ${ }^{18}$ In this study, there was negligible mortality due to LGIB, supporting the previous studies, which could be attributed to younger age of patients and less coexistent comorbid conditions as age is an independent predictor of mortality in patients with LGIB.

\section{Conclusion}

LGIB is fairly a common clinical presentation in many patients presenting with intestinal bleeding and is a disorder in the predominantly younger population. The clinical spectrum of LGIB varies according to age and sex, as well as geographical profile of patients. Colonoscopy is the initial investigation of choice, with a significant diagnostic yield. Hence, LGIB is to be approached taking into account the coexistent comorbidities and geographical and agewise variance in etiology for the better management of patients.

\section{Limitations}

Small intestine could not be evaluated by enteroscopy or capsule endoscopy, as both not available in our institute. Also, the study population was less. 


\section{Conflicts of Interest}

None declared.

\section{References}

1 Strate LL. Lower GI bleeding: epidemiology and diagnosis. Gastroenterol Clin North Am 2005;34(4):643-664

2 Longstreth GF. Epidemiology and outcome of patients hospitalized with acute lower gastrointestinal hemorrhage: a population-based study. Am JGastroenterol 1997;92(3):419-424

3 Peura DA, Lanza FL, Gostout CJ, Foutch PG. The American College of Gastroenterology Bleeding Registry: preliminary findings. Am J Gastroenterol 1997;92(6):924-928

4 Bloomfeld RS, Rockey DC, Shetzline MA. Endoscopic therapy of acute diverticular hemorrhage. Am J Gastroenterol 2001;96(8):2367-2372

5 Farrell JJ, Friedman LS. Review article: the management of lower gastrointestinal bleeding. Aliment Pharmacol Ther 2005;21(11):1281-1298

6 Longstreth GF. Epidemiology of hospitalization for acute upper gastrointestinal hemorrhage: a population-based study. Am J Gastroenterol 1995;90(2):206-210

7 Velayos FS, Williamson A, Sousa KH, et al. Early predictors of severe lower gastrointestinal bleeding and adverse outcomes: a prospective study. Clin Gastroenterol Hepatol 2004;2(6):485-490

8 Al Qahtani AR, Satin R, Stern J, Gordon PH. Investigative modalities for massive lower gastrointestinal bleeding. World J Surg 2002;26(5):620-625
9 Bhasin DK, Rana SS. Lower Gastrointestinal Bleed. Med Update 2011:332- 335

10 Schmulewitz N, Fisher DA, Rockey DC. Early colonoscopy for acute lower GI bleeding predicts shorter hospital stay: a retrospective study of experience in a single center. Gastrointest Endosc 2003;58(6):841-846

11 Jensen DM, Machicado GA. Diagnosis and treatment of severe hematochezia. The role of urgent colonoscopy after purge. Gastroenterology 1988;95(6):1569-1574

12 Boley SJ, DiBiase A, Brandt LJ, Sammartano RJ. Lower intestinal bleeding in the elderly. Am J Surg 1979;137(1):57-64

13 Dar IA, Dar WR, Kasana BA, et al. Etiology, clinical presentation, diagnosis and management of lower gastrointestinal bleed in a tertiary care hospital in India: a retroprospective study. J Dig Endosc 2015;6:101-109

14 Goenka MK, Kochhar R, Mehta SK. Spectrum of lower gastrointestinal hemorrhage: an endoscopic study of 166 patients. Indian J Gastroenterol 1993;12(4):129-131

15 Khandelwal C. Lower gastrointestinal bleeding. Indian J Surg 2003;65:151-155

16 Bai Y, Peng J, Gao J, Zou DW, Li ZS. Epidemiology of lower gastrointestinal bleeding in China: single-center series and systematic analysis of Chinese literature with 53,951 patients. J Gastroenterol Hepatol 2011;26(4):678-682

17 Bokhari M, Vernava AM, Ure T, Longo WE. Diverticular hemorrhage in the elderly-is it well tolerated? Dis Colon Rectum 1996;39(2):191-195

18 Strate LL, Orav EJ, Syngal S. Early predictors of severity in acute lower intestinal tract bleeding. Arch Intern Med 2003;163(7):838-843 